\title{
Do subcritical bubbles hinder first order phase transition?
}

\author{
KARI ENQVIST ${ }^{1}$ \\ Department of Physics, P.O. Box 9, FIN-00014 University of Helsinki, Finland \\ Antonio Riotto ${ }^{2}$ \\ International School for Advanced Studies, SISSA-ISAS \\ Starda Costiera 11, I-34014, Miramare, Trieste, Italy \\ and \\ Istituto Nazionale di Fisica Nucleare, Sezione di Padova, 35100 Padova, Italy \\ IIRO VILJA ${ }^{3}$ \\ Department of Physics, University of Turku, FIN-20500 Turku, Finland
}

May 16, 2021

\begin{abstract}
We consider the role played by subcritical bubbles during the electroweak phase transition, estimate their average size, amplitude and formation rate taking into account the crucial role played by thermalization. We also study the influence of subcritical bubbles on the formation of critical bubbles in the thin wall regime and show that, contrary to some recent claims, subcritical bubbles do not affect the nucleation of critical bubbles in an appreciable way. From this fact we conclude that the electroweak baryogenesis scenarios associated with a first order electroweak phase transition still remain an attractive possibility.
\end{abstract}

\footnotetext{
1enqvist@phcu.helsinki.fi; ${ }^{2}$ riotto@tsmi19.sissa.it. Address after November 95: Theoretical Astrophysics Group, NASA/Fermilab, Batavia, IL60510, USA.; ${ }^{3}$ vilja@utu.fi
} 


\section{Introduction}

Critical bubble nucleation during a first order electroweak phase transition has received much attention since the discovery of the possibility for electroweak baryogenesis [1]. Indeed, one of the basic ingredients for the generation of the baryon asymmetry (apart from the requirement of baryon- and CP-violating interactions) is the presence of an out-of-equilibrium state [2] which, during the first order electroweak phase transition with supercooling, is attained by critical bubbles expanding in the thermal bath of the unbroken phase.

Less attention has been paid to the environment where the critical bubble nucleation is supposed to occur. Since critical bubbles have a finite size, phase transitions are highly local phenomena. Fluctuations with spatial correlations comparable to the critical bubble size may be expected to be important for bubble nucleation. Also, if thermal fluctuations are too large, any perturbative scheme could break down. In such a situation prediction of a first order phase transition becomes suspect, with the possibility that the entire scenario of electroweak baryogenesis might be invalidated.

Although the presence of thermal fluctuations in any hot system is undisputed, their role in the dynamics of weakly first order phase transitions is still under debate [3].

The idea that statistical fluctuations around equilibrium are spherically symmetric and have roughly a correlation volume, where the correlation length is given by the inverse temperature dependent mass of the Higgs field, $\xi(T)=m^{-1}(T)$, was first discussed in [1]. These fluctuations are referred to as subcritical bubbles. The amplitude of the thermal fluctuations was estimated in ref. [4], where it was concluded that they are dominant if the Higgs mass $m_{H}$ is larger than $\sim 80 \mathrm{GeV}$, whence the fraction which the asymmetric vacuum occupies in the neighborhood of the critical temperature becomes of the order of unity. Therefore it was concluded that critical bubbles cannot be generated due to the inhomogeneities of the background field. In ref. [4], however, the continuous disappearance of the subcritical bubbles was not accounted for. This can happen in two ways: the subcritical bubbles, being unstable configurations, tend to shrink; the bubbles are also subject of constant thermal bombardment so that they may disappear simply because of thermal noise. The thermalization rate of small-amplitude configurations near the critical temperature has been estimated in ref. [5 for the electroweak phase transition, and it was found that, compared with typical first order transition times, thermalization is rather fast.

Kinetics of subcritical bubbles has been investigated by Gelmini and Gleiser [6], who found, under a specific assumption about the form of the destruction rate due to thermal noise, that for Higgs masses below $\sim 55 \mathrm{GeV}$ the approach to equilibrium is dominated by shrinking. Unfortunately, for the interesting range of Higgs masses 
dictated by the experimental constraints coming from LEP, $m_{H}>60 \mathrm{GeV}$ [7], their analysis is inconclusive since the approximations adopted break down. Nucleation induced by the growth of conglomerates of subcritical bubbles has been discussed in [8].

Very recently, Shiromizu et al. [9] have re-estimated the amplitude of thermal fluctuations by calculating the typical size of subcritical bubbles during the electroweak phase transition. They claim that for any experimentally allowed values of the Higgs mass, the amplitude of thermal fluctuations always exceeds the first reflection point of the effective potential. From this analysis they conclude that any standard electroweak baryogenesis scenario associated with a first order first transition cannot work.

Their starting point is the observation that at the microscopic level, the true origin of the dominant thermal fluctuation is the perpetual creation and annihilation of spherical subcritical bubbles. Thus one should identify the typical size $R$ of the bubbles with the size estimated by a statistical ensemble averaging, instead of assuming it to be the correlation length. Since the amplitude of the thermal fluctuations sensitively depends on the size of the subcritical bubble, a small change in the size results in a drastic change in the details of the phase transition. Indeed, modeling any subcritical bubbles with a Gaussian shape, the authors of ref. [9] find that the typical size $\langle R\rangle$ of a subcritical bubble is smaller than the correlation length, and that the amplitude of thermal fluctuations increases compared with the previous estimates.

In the present paper we wish to critically reanalyze the results of ref. [9]. We reconsider the role played by the subcritical bubbles during the electroweak phase transition, estimate their size, amplitude and the formation rate. Moreover, we study the influence of subcritical bubbles on the formation of a critical bubble by comparing the average subcritical energy density associated with the fluctuation to the energetics of barrier penetration in the thin wall approximation. Our final conclusion, which differs from ref. [9], is that subcritical bubbles do not affect nucleation of critical bubbles in an appreciable way.

The paper is organized as follows. In Sect. 2 we briefly describe the small supercooling limit and the thin wall approximation. In Sect. 3 we discuss the salient features of subcritical fluctuations and present our Ansatz subcritical bubbles. Thermal averages are performed in Sect. 4, and the crucial role of thermalization is discussed in Sect. 5. Finally, our numerical results and conclusions are given in Sect. 6 .

\section{Small supercooling limit and thin wall approxi- mation}

First order phase transition and bubble dynamics in the Standard Model have lately been studied in much detail, and it has become increasingly clear [10] that for Higgs 
masses considerably heavier than $60 \mathrm{GeV}$, the electroweak phase transition is only of weakly first order. For Higgs mass $m_{H}>100 \mathrm{GeV}$ the calculations, both perturbative and lattice ones, confront technical problems a phenomenological Higgs potential for the order parameter $\phi$ suitable for a simple description of a first order phase transition:

$$
V(\phi)=\frac{1}{2} m^{2}(T) \phi^{2}-\frac{1}{3} \alpha T \phi^{3}+\frac{1}{4} \lambda \phi^{4}
$$

where we have not determined the parameters perturbatively but fit them, when needed, according to a recent two-loop calculation of the gauge invariant effective potential [11]. Most of the dynamical properties of the electroweak phase transition associated with the potential Eq. (1), such as the smallness of the latent heat, the bubble nucleation rate and the size of critical bubbles, have been discussed in [12]. For the purposes of the present paper it suffices to recall only some of the results.

First we need the size of the critical bubble. Assuming that there is only little supercooling, as seems to be the case for the electroweak phase transition, the bounce action can be written as

$$
S / T=\frac{\alpha}{\lambda^{3 / 2}} \frac{2^{9 / 2} \pi}{3^{5}} \frac{\bar{\lambda}^{3 / 2}}{(\bar{\lambda}-1)^{2}},
$$

where $\bar{\lambda}(T)=9 \lambda m^{2}(T) /\left(2 \alpha^{2} T^{2}\right)$. The cosmological transition temperature is determined from the relation that the Hubble rate equals the transition rate $\propto e^{-S / T}$, yielding $S / T_{f} \simeq \ln \left(M_{P l}^{4} / T_{f}^{4}\right) \simeq 150$, where $T_{f}$ is the transition temperature. Thus we obtain from Eq. (2)

$$
\bar{\lambda}\left(T_{f}\right) \simeq 1-0.0442 \frac{\alpha^{1 / 2}}{\lambda^{3 / 4}} \equiv 1-\delta
$$

On the other hand, small supercooling implies that $1-\bar{\lambda}=\delta \ll 1$, i.e. $\alpha \ll 500 \lambda^{3 / 2}$. Solving for $\bar{\lambda}$ in Eq. (2) yields the transition temperature $T_{f}$. One finds

$$
m^{2}\left(T_{f}\right)=\frac{2 \alpha^{2}}{9 \lambda} \bar{\lambda}\left(T_{f}\right) T_{f}^{2}
$$

The extrema of the potential are given by

$$
\phi_{ \pm}(T)=\frac{\alpha T}{2 \lambda}(1 \pm \sqrt{1-8 \bar{\lambda} / 9}) .
$$

Expanding the potential at the broken minimum $\phi_{+}(T)$ we find

$$
-\epsilon \equiv V\left(\phi_{+}, T_{f}\right)=\frac{1}{6} m^{2}\left(T_{f}\right) \phi_{+}^{2}-\frac{1}{12} \lambda \phi_{+}^{4}=-0.00218 \frac{\alpha^{9 / 2}}{\lambda^{15 / 4}} T_{f}^{4}+\mathcal{O}\left(\delta^{2}\right) .
$$

\footnotetext{
${ }^{1}$ It is conceivable that for such large Higgs masses the electroweak phase transition is close to a second order and does not proceed by critical bubble formation.
} 
The height of the barrier is situated at $\phi_{-} \simeq \phi_{+} / 2$ with $V\left(\phi_{-}, T_{c}\right) \equiv V_{\max }=\alpha^{4} T_{c}^{4} /\left(144 \lambda^{3}\right)$, where $T_{c}$ is the temperature at which $V(0)=V\left(\phi_{+}\right)$, given by the condition $m\left(T_{c}\right)^{2}=$ $\left(2 \alpha^{2} T_{c}^{2} / 9 \lambda\right)$. As $T_{c} \simeq T_{f}$ we may conclude that the thin wall approximation is valid if $-\epsilon / V_{\max }=0.314 \alpha^{1 / 2} / \lambda^{3 / 4} \ll 1$, or $\alpha \ll 10 \lambda^{3 / 2}$. Thus the small supercooling limit is clearly satisfied if the thin wall approximation is valid.

To get the size of the critical bubble we still need the surface tension. One easily finds

$$
\sigma=\int_{0}^{\infty} d \phi \sqrt{2 V\left(T_{c}\right)}=\frac{2 \sqrt{2} \alpha^{3}}{91 \lambda^{5 / 2}} T_{c}^{3}
$$

We define the critical bubble radius by extremizing the bounce action. The result is

$$
R_{c}=13.4 \frac{\lambda^{3 / 4}}{\alpha^{1 / 2} m\left(T_{f}\right)} .
$$

Therefore $R_{c}$ is much larger than the correlation length $\xi\left(T_{f}\right)=1 / m\left(T_{f}\right)$ at the transition temperature, as it should.

\section{$3 \quad$ Subcritical bubbles}

We begin this Section by briefly describing the procedure adopted in ref. [9]. The following spherical Ansatz was chosen for the subcritical bubble configuration:

$$
\phi_{g}(r)=\phi_{+} \exp \left[-\frac{r^{2}}{R(t)^{2}}\right]
$$

where $R(t)$ is the time dependent size of the subcritical bubble and $r$ is the radial coordinate. In order to estimate the typical size of a subcritical bubble, one defines the canonical momentum $p_{R} \equiv \partial L_{\text {eff }}(\dot{R}, R) / \partial \dot{R}$, whence an effective Hamiltonian

$$
H_{\mathrm{eff}}\left(R, p_{R}\right) \equiv p_{R} \dot{R}-L_{\mathrm{eff}}
$$

can be constructed.

The thermal average of $R$ was defined as

$$
\langle R\rangle=\frac{\int d p_{R} d R R e^{-\beta H_{\mathrm{eff}}}}{\int d p_{R} d R e^{-\beta H_{\mathrm{eff}}}},
$$

which, in the neighborhood of the critical temperature, turns out to be much smaller than $\xi\left(T_{c}\right)$.

To find the thermal fluctuation amplitude on the scale $\langle R\rangle$, the following trial function was adopted

$$
\phi(r)=a \exp \left[-\frac{r^{2}}{\langle R\rangle^{2}}\right]
$$


whence the amplitude in the symmetric vacuum was given by

$$
\left\langle a^{2}\right\rangle=\frac{\int d a a^{2} e^{-\beta F(a, T)}}{\int d a e^{-\beta F(a, T)}} .
$$

At $T \simeq T_{c}$ the amplitude of a fluctuation $\sqrt{\left\langle a^{2}\right\rangle}$ exceeded the inflection point $\phi_{-}\left(T_{c}\right)$ and from this fact it was concluded that thermal fluctuations drastically change the dynamics of the phase transition from the ordinary first order type with supercooling.

We now wish to make some critical comments on the approach used in ref. [9] and present a remedy to it, which, as we shall argue, is more realistic.

Let us first make the general observation that it is the actual transition temperature $T_{f}$ rather than the critical temperature $T_{c}$ which is relevant for the study on subcritical bubbles. This is true in the sense that if critical bubbles are not important at $T_{f}$, they most certainly will not be so at $T_{c}$. As we shall show, it actually turns out that subcritical bubbles are not important even at $T_{f}$. This justifies, in retrospect, our choice $T=T_{f}$ for performing the calculations.

In the case of a weak first order phase transition the critical bubble is typically well described by a thin wall approximation, where the configuration is by no means gaussian, but has a flat 'highland' (with $\phi$ determined by the non-zero minimum of the potential) and a steep slope down to $\phi=0$. Therefore it seems natural that also a large subcritical bubble should resemble the critical one, i.e. when $R$ increases, the form of the subcritical bubble should deform smoothly so that, when $R=R_{c}$, the bubble is a critical one. This behaviour is not reproduced by the Ansatz of Shiromitzu et al. , given in Eq. (9). Moreover, it has the strange property that in the limit $R \rightarrow 0$, the bubble becomes infinitely sharp because the amplitude of the fluctuation is kept fixed, while, in general, one would expect that the amplitude of the fluctuations depends sensitively on their spatial size. Finally, but no less important, the calculation of $\sqrt{\left\langle a^{2}\right\rangle}$, Eq. (13), is performed by substituting for the typical size of the subcritical bubble the average $\langle R\rangle$ as calculated from Eq. (11), which only is valid for a particular value of $a$, namely $a=\phi_{+}$. In other words, in ref. [9] the only statistical degree of freedom is taken to be the size of subcritical bubbles.

Motivated by these observations, let us define a subcritical bubble as a functional of both the amplitude $a$ and the radius $R$. For this purpose one has first to study the behaviour of the potential as a function of the amplitude. At $T_{f}$ there is a interval $\phi \in\left[a_{-}, a_{+}\right]$where $V(\phi) \leq 0$. If the amplitude of the bubble is in that interval, there exists a critical bubble-solution of the bounce action. This means that we have a relation $R_{c}=R_{c}(a)$ which reproduces Eq. (8) if $a=\phi_{+}$. Therefore $R_{c}(a)$ serves as an upper limit for the bubble radius in that region.

Moreover, as argued above, the subcritical bubble should be of the thin wall type, with the central region having the field value somewhere in the interval $\phi \in\left[a_{-}, a_{+}\right]$. 
These considerations lead us to define different Ansätze for various regions in the $(a, R)$-plane. When $\phi \in\left[a_{-}, a_{+}\right]$, we use an Ansatz such that when $R \rightarrow R_{c}(a)$, the field configuration goes towards the thin wall form. For small $R$ we use a simple gaussian configuration. For other values of $a$ we always take a thin-wall like Ansatz. Thus we write for $\phi \in\left[a_{-}, a_{+}\right]$and $R \leq R_{c}(a)$

$$
\phi(t, R)=a(t)\left[\frac{R_{c}-R}{R_{c}} \phi_{g}+\frac{R}{R_{c}} \phi_{t}\right]
$$

where $t$ is the time coordinatet and

$$
\begin{aligned}
& \phi_{g}(R)=e^{-r^{2} / R^{2}}, \\
& \phi_{t}(R)=1 /\left(e^{m(r-R)}+1\right) .
\end{aligned}
$$

Such an Ansatz reproduces the requirement that when $R \rightarrow R_{c}$, subcritical bubbles should resemble critical ones. In practise the statistical averages depend only weakly on $a$ because the main contribution to them comes from the region of small $a$ and large $R$. Therefore we assume for simplicity that criticality depends only weakly on $a$ and take $R_{c}(a)=R_{c}$ to be a constant whenever possible.

For $\phi \notin\left[a_{-}, a_{+}\right]$we assume that no gaussian component is present and write simply

$$
\phi(t, R)=a(t) \phi_{t}(R)
$$

However, the statistical averages are excepted to be quite insensitive of the precise form of the bubble.

These Ansätze can be plugged into the action

$$
S[a, R]=\int d^{4} x\left[\frac{1}{2}(\partial \phi)^{2}-V(\phi)\right]
$$

from which the Lagrangian in terms of the dynamical variables $a$ and $R$ can be extracted. In the practical calculation we have, whenever possible, approximated $\phi_{t}$ by the step function. After that is a simple matter to calculate the effective Hamiltonian function $H_{\text {eff }}$ of the dynamical variables $a$ and $R$.

\section{Statistical averages}

Once we have the Hamiltonian, we may calculate the statistical average of a dynamical variable of the type $F(a, R)$ simply by

$$
\langle F(a, R)\rangle=\frac{\int d p_{R} d p_{a} d a d R F(a, R) e^{-\beta H_{\mathrm{eff}}}}{\int d p_{R} d p_{a} d a d R e^{-\beta H_{\mathrm{eff}}}} .
$$

\footnotetext{
${ }^{2}$ Note, however, that we need not to specify the explicit time evolution of $a$ and $R$ when dealing with statistical averages.
} 
However, because the effective Lagrangian is of the form

$$
\mathcal{L}_{\text {eff }}=\frac{1}{2}\left(\begin{array}{ll}
\dot{a} & \dot{R}
\end{array}\right) K\left(\begin{array}{c}
\dot{a} \\
\dot{R}
\end{array}\right)-\mathcal{V},
$$

where $K=K(a, R)$ is a symmetric matrix, after the momentum integration the average can be cast into the form

$$
\langle F(a, R)\rangle=\frac{\int d a d R F(a, R) \sqrt{\operatorname{det} K} e^{-\beta \mathcal{V}}}{\int d a d R \sqrt{\operatorname{det} K} e^{-\beta \mathcal{V}}} .
$$

The matrix

$$
K=4 \pi\left(\begin{array}{ll}
K_{11} & K_{12} \\
K_{21} & K_{22}
\end{array}\right)
$$

and the pseudopotential $\mathcal{V}$ are given separately for the two regions. For $\phi \in\left[a_{-}, a_{+}\right]$ we obtain

$$
\begin{aligned}
R_{c}^{2} K_{11}= & \Delta^{2} R^{3} A_{2}^{2}+2 \Delta R^{4} B_{2}^{1}+\frac{1}{3} R^{5} \\
R_{c}^{2} K_{12}= & 2 a \Delta^{2} R^{2} A_{4}^{2}-a \Delta R^{3} A_{2}^{2}+\frac{1}{3} a R^{4}+a R^{5} m I(m R) \\
& +a \Delta R^{3} B_{2}^{1}-a R^{4} B_{2}^{1}+2 a \Delta R^{3} B_{4}^{1}+a \Delta R^{4} m J_{2}(m R) \\
R_{c}^{2} K_{22}= & 4 a^{2} \Delta^{2} R A_{6}^{2}+a^{2} R^{5} m^{2} I(2 m R)+4 a^{2} \Delta R^{3} m J_{4}(m R) \\
& +a^{2} R^{3} A_{2}^{2}-2 a^{2} R^{3} B_{2}^{1}+\frac{1}{3} a^{2} R^{3}-a^{2} \Delta R^{2} A_{4}^{2} \\
& -2 a^{2} R^{4} m J_{2}(m R)+4 a^{2} \Delta R^{2} B_{4}^{1}+2 a^{2} R^{4} m I(m R)
\end{aligned}
$$

and

$$
\begin{aligned}
\frac{R_{c}(a)^{2}}{4 \pi} \mathcal{V}= & 2 a^{2} \Delta^{2} R A_{4}^{2}+2 a^{2} \Delta R^{3} m J_{3}(m R)+\frac{1}{2} a^{2} R^{5} m^{2} I(2 m R) \\
& +\frac{1}{2} m^{2} a^{2} \Delta^{2} R^{3} A_{2}^{2}+m^{2} a^{2} \Delta R^{4} B_{2}^{1}+\frac{1}{6} m^{2} a^{2} R^{5}-\frac{1}{3} \alpha T \frac{a^{3}}{R_{c}(a)} \Delta^{3} R^{3} A_{2}^{3} \\
& -\alpha T \frac{a^{3}}{R_{c}(a)} \Delta^{2} R^{4} B_{2}^{2}-\alpha T \frac{a^{3}}{R_{c}(a)} \Delta R^{5} B_{2}^{1}-\frac{1}{9} \alpha T \frac{a^{3}}{R_{c}(a)} R^{6} \\
& +\frac{1}{4} \lambda \frac{a^{4}}{R_{c}(a)^{2}} \Delta^{4} R^{3} A_{2}^{4}+\lambda \frac{a^{4}}{R_{c}(a)^{2}} \Delta^{3} R^{4} B_{2}^{3}+\frac{3}{2} \lambda \frac{a^{4}}{R_{c}(a)^{2}} \Delta^{2} R^{5} B_{2}^{2} \\
& +\lambda \frac{a^{4}}{R_{c}(a)^{2}} \Delta R^{6} B_{2}^{1}+\frac{1}{12} \lambda \frac{a^{4}}{R_{c}(a)^{2}} R^{7} .
\end{aligned}
$$

Note that in Eq. (24) the $a$-dependence of $R_{c}$ has to be used explicitly because the critical behavour is determined from it. For the region where $\phi \notin\left[a_{-}, a_{+}\right]$the corresponding functions are given by

$$
\begin{aligned}
K_{11} & =\frac{1}{3} R^{3} \\
K_{12} & =a R^{3} m I(m R) \\
K_{22} & =a^{2} R^{3} m^{2} I(2 m R)
\end{aligned}
$$


and

$$
\frac{1}{4 \pi} \mathcal{V}=\frac{1}{2} a^{2} R^{3} m^{2} I(2 m R)+\frac{1}{6} m^{2} a^{2} R^{3}-\frac{1}{9} \alpha T a^{3} R^{3}+\frac{1}{12} \lambda a^{4} R^{3}
$$

A number of shorthand notations have been introduced in the previous equations:

$$
\begin{aligned}
\Delta & =R_{c}(a)-R \\
A_{n}^{k} & =\int_{0}^{\infty} d u u^{n} e^{-k u^{2}}=\frac{\Gamma\left(\frac{n+1}{2}\right)}{2 k^{\frac{n+1}{2}}} \\
B_{n}^{k} & =\int_{0}^{1} d u u^{n} e^{-k u^{2}} \\
I(x) & =\int_{0}^{1} d u u^{2} e^{x(u-1)}=\frac{1}{x}-\frac{2}{x^{2}}+\frac{2}{x^{3}}-\frac{2}{x^{3}} e^{-x} \\
J_{n}(x) & =\int_{0}^{1} d u u^{n} e^{-u^{2}+x(u-1)} .
\end{aligned}
$$

\section{Thermalization}

Motivated by the fact that thermal fluctuations can generate configurations with spatial size comparable to the critical bubble radius, which may affect the dynamics of a first order phase transition, the authors of ref. [5] have estimated the lifetime of fluctuations of an on-shell Higgs field with zero momentum $\left(p_{0}=m(T), \mathbf{p}=0\right)$. This choice reflects the fact that critical bubbles are typically much larger than the interparticle distance $\simeq 1 / T$ in plasma. Writing $p_{0} \equiv \omega-i \gamma / 2$, one finds that the dispersion relation is

$$
\omega^{2}=|\mathbf{p}|^{2}+m^{2}(T)+\frac{1}{4} \gamma^{2}
$$

where

$$
\gamma=\frac{\operatorname{Im} \Gamma^{(2)}}{\omega}
$$

$\Gamma^{(2)}$ being the two-point function for the Higgs field.

The imaginary part arises at one loop level, but because of kinematical constraints, the two loop contribution is actually dominant in the region of physical couplings. The thermalization rate $\gamma$ for small amplitude scalar fluctuations and large spatial size, $R \sim|\mathbf{p}|^{-1} \gg \gamma^{-1}$, is estimated [5] to be of the order $\gamma \simeq 10^{-2} T$ near the critical temperature, i.e. much larger than the typical first order transition time. This means that all small amplitude fluctuations with size larger than

$$
R_{\max }=\mathcal{O}(1 / \gamma)
$$

will effectively be absent from the mixture of subcritical bubbles and must not counted in the thermal averages. In practise, the limit Eq. (34) is of the order of few times $R_{c}$, depending on the actual value of $\gamma$. Even if it is not precisely known, its inclusion in 
the calculations is important. Without it all statistical averages would be dominated by infinite, infinitesimally small fluctuations. Technically this can be seen from the Eq. (21), where the integrals diverge in the limit $a \rightarrow 0, R \rightarrow \infty$. It is important to note that the divergence is not a problem of our Ansatz but merely a more general phenomenon, which seems to be related to the general infra-red instability problems emerging in the calculations of the effective action.

\section{$6 \quad$ Results and conclusions}

We have computed the average radius and the amplitude of fluctuations at $T=T_{f}$ from Eq. (21) numerically, using a cut-off $R_{\max }$ as discussed in the previous Section. For definiteness, we fitted our phenomenological potential Eq. (1) to the two loop result for the effective potential calculated in [11] for the Higgs mass $M_{H}=70 \mathrm{GeV}$. This yields $\alpha \simeq 0.048$ and $\lambda \simeq 0.061$. One readily verifies that we are indeed safely in the thin wall limit. With these parameter values, in units of $R_{c}, T_{f}=85.70, m^{2}\left(T_{f}\right)=56.78$ and $\gamma \simeq 10^{-2} T_{f} \simeq 0.875$.

Choosing, for instance, $R_{\max } \simeq 6.6 R_{c}$, we find (in units of $R_{c}$ )

$$
\langle a\rangle=1.16 ; \quad\langle R\rangle=2.93 ; \quad\left\langle a^{2}\right\rangle=12.6 ; \quad\left\langle R^{2}\right\rangle=12.2 \text {. }
$$

Note that $\xi=m^{-1}\left(T_{f}\right) \ll\langle R\rangle$, so that the average subcritical bubbles are much larger than the correlation size, as seems reasonable. Indeed, the correlation length gives the order of magnitude of the size of a (thermal) quantum fluctuation, so that, since to build up a bubble configuration many quanta are needed, one can expect that $\langle R\rangle$ is larger than $\xi\left(T_{f}\right)$. Here we remind the reader that the authors of ref. 99 find the opposite result, which is somehow suspect when the above considerations are taken into account.

As far as the production rate for subcritical bubbles is concerned, it is roughly given by 4

$$
\Gamma_{s c} \simeq T_{f} \exp \left(-S / T_{f}\right)
$$

where $S$, given by Eq. (18), is the free energy for the subcritical configuration.

We have found the average rate for subcritical bubble formation to be much larger than the Hubble rate $H$ :

$$
\Gamma_{s c}=T_{f}\left\langle e^{-S / T}\right\rangle \simeq 0.49 T_{f} \gg H
$$

Thus we find out that the subcritical bubbling is so fast that effectively the background, on which a critical bubble is to formed, is filled by subcritical fluctuations. In principle, this could affect the way by which critical bubbles are produced and naively, one could think that the extra energy would facilitate barrier penetration or possibly even 
invalidate our thin wall approach. That this is not the case can be ascertained by considering the average fluctuation energy about $\phi=0$. Using Eq. (35) one obtains

$$
\langle V(a)\rangle \simeq \frac{1}{2} m^{2}\left(T_{f}\right)\left\langle a^{2}\right\rangle=357.7 \ll-\epsilon \simeq 5 \times 10^{3},
$$

where Eq. (6) and the fact that $\phi_{+} \simeq 48.05$ have been used. Also the average amplitude is clearly smaller than the inflection point, $\sqrt{\left\langle a^{2}\right\rangle} \ll \phi_{-}$. Thus, in terms of barrier penetration, subcritical fluctuations represent only a minor correction and they have no remarkable effect on the thin wall approximation.

We should point out that the results Eq. (35) are rather sensitive to $R_{\text {max }}$; for instance, if we took $R_{\max } \simeq 3.3 R_{c}$, we would gotten $\langle a\rangle=1.94,\langle R\rangle=1.53$, together with an almost twice as large $\left\langle a^{2}\right\rangle$ and an $\left\langle R^{2}\right\rangle$ smaller by a factor of about four. This is a general trend: as $R_{\max }$ increases, $\langle a\rangle$ and $\left\langle a^{2}\right\rangle$ decrease so that the amplitude becomes more peaked, while $\langle R\rangle$ and $\left\langle R^{2}\right\rangle$ grow. To explain analytically, for instance, the scaling of $\langle R\rangle$ with the changing of $R_{\max }$ one can consider the limit $R_{c} \rightarrow \infty$ in Eq. (21) which is reasonable in the vicinity of $T_{c}$. In such a case, it is easy to show that

$$
\langle R\rangle \propto \frac{\int_{0}^{R \max } d R R^{1 / 2}}{\int_{0}^{R \max } d R R^{-1 / 2}}=R_{\max },
$$

whereas a similar calculation proves that $\left\langle R^{2}\right\rangle \propto R_{\max }^{2}$. It is then clear that why, increasing $R_{\max }$ by a factor two, $\langle R\rangle$ and $\langle R\rangle^{2}$ increase by a factor two and four, respectively. Analogous considerations explain the decreasing of $\langle a\rangle$ and $\left\langle a^{2}\right\rangle$ with increasing $R_{\max }$.

Note, however, that, for reasonable values of the cut-off $R_{\max }$, the subcritical nucleation rate does not change, nor the conclusion that $\langle V(a)\rangle \ll-\epsilon$.

To conclude, we have find that subcritical bubbles are not, at least for Higgs masses less than about $100 \mathrm{GeV}$, important during the onset of the electroweak phase transition. We have argued that critical bubble formation proceeds essentially unchanged, and here we disagree with the result of Shiromizu et al. [9]. The main reason for this discrepancy is that in ref. [9] the only statistical degree of freedom describing the properties of subcritical bubbles is taken to be their size $R$, keeping their amplitude fixed at the value $\phi_{+}$. This fact led the authors of ref. [9] to conclude that $\langle R\rangle \ll \xi\left(T_{c}\right)$ which appears not reasonable. Indeed, to build up a classical configuration, many thermal quanta (whose typical size is the correlation length) are needed and one would then expect that $\langle R\rangle \gg \xi\left(T_{c}\right)$. To recover such a behaviour, we have treated both the amplitude and the critical size of subcritical bubbles as statistical degrees of freedom. We have then estimated their average size, amplitude and formation rate taking into account the crucial role played by thermalization, showing that subcritical bubbles do not affect the nucleation of critical bubbles in an appreciable way. From this fact we 
conclude that the electroweak baryogenesis scenarios associated with a first order electroweak phase transition remain a viable possibility to explain the primordial baryon asymmetry in the Universe. 


\section{References}

[1] For a review, see A.G. Cohen, D.B. Kaplan and A.E. Nelson, Ann. Rev. Nucl. Part. Phys. 43, 27 (1993) ; D.B. Kaplan, contribution to the $4^{\text {th }}$ International Conference on Physics Beyond the Standard Model, Lake Tahoe, 13-16 December 1994, hepph 9503360.

[2] A.D. Sakharov, Pis'ma Zh. Eksp. Fiz. 5, 32 (1967); JETP Lett. 5, 24 (1967).

[3] M. Dine, R. Leigh, P. Huet, A. Linde and D. Linde, Phys. Rev. D46, 550 (1992); G. Anderson, Phys. Lett. B295, 32 (1992).

[4] M. Gleiser, E.W. Kolb and R. Watkins, Nucl. Phys. B364, 411 (1991); M. Gleiser and E.W. Kolb, Phys. Rev. Lett. 69, 1304 (1992); Phys. Rev. D48, 1560 (1993); M. Gleiser and R.O. Ramos, Phys. Lett. B300, 271 (1993); N. Tetradis, Z. Phys. C57, 331 (1993).

[5] P. Elmfors, K. Enqvist and I. Vilja, Nucl. Phys. B412, 459 (1994).

[6] G. Gelmini and M. Gleiser, Nucl. Phys. B419, 459 (1994).

[7] Particle Data Group, Review of Particle Properties, Phys. Rev. D50, 1173 (1994).

[8] K. Enqvist and I. Vilja, Phys. Lett. B344, 98 (1995).

[9] T. Shiromizu, M. Morikawa and J. Yokoyama, Preprint YITP/U-95-1, KUNS1297, OCHA-PP-51 (1995).

[10] K. Kajantie, K. Rummukainen and M. Shaposnikov, Nucl. Phys. B407, 27 (1993); K. Farakos, K. Kajantie, K. Rummukainen and M.E. Shaposhnikov, Phys. Lett. B336, 494 (1994); B. Bunk, E.M. Ilgenfriz, J. Kripfganz and A. Schiller, Nucl. Phys. B403, 453 (1993); F. Csikor et al., Phys. Lett. B334, 405 (1994).

[11] Z. Fodor and Hebecker, Nucl. Phys. B432, 127 (1994).

[12] K. Enqvist, J. Ignatius, K. Kajantie and K. Rummukainen, Phys. Rev. D45, 3415 (1992). 